\title{
POUR COMPRENDRE LES NOUVELLES LIAISONS DIGITALES : LE CONCEPT D'INDIVIDUATION CHEZ CARL JUNG ET GILBERT SIMONDON
}

José Pinheiro Neves

De Boeck Supérieur | «Sociétés »

2011/1 n $111 \mid$ pages 105 à 114

ISSN 0765-3697

ISBN 9782804165413

Article disponible en ligne à l'adresse :

http://www.cairn.info/revue-societes-2011-1-page-105.htm

\section{!Pour citer cet article :}

José Pinheiro Neves, « Pour comprendre les nouvelles liaisons digitales : le concept d'individuation chez Carl Jung et Gilbert Simondon », Sociétés 2011/1 (n¹11), p. 105-114. DOI $10.3917 /$ soc. 111.0105

Distribution électronique Cairn.info pour De Boeck Supérieur.

(C) De Boeck Supérieur. Tous droits réservés pour tous pays.

La reproduction ou représentation de cet article, notamment par photocopie, n'est autorisée que dans les limites des conditions générales d'utilisation du site ou, le cas échéant, des conditions générales de la licence souscrite par votre établissement. Toute autre reproduction ou représentation, en tout ou partie, sous quelque forme et de quelque manière que ce soit, est interdite sauf accord préalable et écrit de l'éditeur, en dehors des cas prévus par la législation en vigueur en France. Il est précisé que son stockage dans une base de données est également interdit. 


\title{
POUR COMPRENDRE LES NOUVELLES LLAISONS DIGITALES : LE CONCEPT D'INDIVIDUATION CHEZ CARL JUNG ET GILBERT SIMONDON
}

José Pinheiro NEVES *

\begin{abstract}
Résumé : Dans ce texte, nous irons voir les similitudes de Carl Jung et Gilbert Simondon par rapport à la façon de penser le concept d'individuation. Les contributions de ces deux auteurs permettent justement de repenser les formes d'imaginaire dans la société postmoderne pour une meilleure compréhension des nouvelles liaisons (ou médiations socio-digitales) humaines. À la fin du texte, nous suggérons certains exemples d'application du concept d'individuation.
\end{abstract}

Mots clés : individuation, technique, Simondon, Jung.

Abstract : In this essay, we'll see the similarities between Carl Jung and Gilbert Simondon about the concept of individuation. The contributions of these two authors allowed me to rethink the forms of imagination in post-modern society for a better understanding of new connections (or mediations socio-digital) between humans. At the end of the text, I suggest some examples of application of the concept of individuation.

Keywords : individuation, technical, Simondon, Jung.

"L'individu moderne, acteur de l'Histoire, est un, il est indivisible. La personne postmoderne, confrontée au destin, ne peut pas être limitée au petit je produit par la crispation égocentrique qui fut la marque des deux ou trois siècles qui viennent de s'écouler. Peut-être fautil à cet égard être attentif à l'enseignement de C.G. Jung et au processus d'"individuation" qu'il analyse. »

Michel Maffesoli, L'instant éternel

Dans ce texte, nous irons voir les similitudes de Carl Jung et Gilbert Simondon par rapport à la façon de penser le concept d'individuation ${ }^{1}$. Les contributions de ces

* Centre d'Études en Communication et Société - Université du Minho, Braga, Portugal, jpneves@ics.uminho.pt

1. Cet article s'appuie sur les travaux réalisés en collaboration avec Pedro Rodrigues Costa (Neves et Costa, 2009). L'auteur remercie aussi Albertino Gonçalves et Jean-Martin Rabot pour leur aide et leurs contributions. 
deux auteurs permettent justement de repenser les formes d'imaginaire dans la société postmoderne pour une meilleure compréhension des nouvelles liaisons (ou médiations socio-digitales) humaines. À la fin du texte, nous suggérons certains exemples d'application du concept d'individuation.

\section{Carl Gustav Jung et le processus d'individuation}

Le concept d'individuation apparaît en 1916 et marque, d'une certaine manière, la rupture de Jung avec le modèle d'analyse de Freud qui était centré sur l'adaptation normale de l'individu. Selon une définition simple, l' individuation » est " un processus par lequel une personne devient consciente de son individualité » 2 . Cette définition, provenant de Carl Gustav Jung, suggère que l'individuation est un processus à travers lequel l'être humain évolue d'un état infantile d'identification totale vers un état de plus grande différenciation, impliquant une ampliation de la conscience et articulant, de manière harmonieuse, ses différentes strates. Par ce processus d'individuation, l'individu s'identifie davantage avec les orientations qui viennent du «Soi-même » - vulgairement défini par l'archétype du Self, c'està-dire la totalité de la personnalité individuelle - qu'avec les comportements, les orientations et les valeurs qui émanent de l'environnement social entourant et qui sont au fondement d'une vision partielle fondée dans la " persona » (Jung, 1984, pp. 35-36). Lorsque nous oublions cette complexité grâce à une socialisation forte, on a tendance à oublier notre côté sombre et à trouver une normalisation autour d'un individu cohérent, autour de la « persona ».

Carl Jung suggère, ainsi, aussi bien une adaptation aux conditions extérieures, surtout aux conditions naturelles, culturelles et sociales, qu'une adaptation aux conditions intérieures comme, par exemple, les perceptions inconscientes et les désirs. Atteindre cette double adaptation n'est pas facile mais aide à obtenir la conscience totale du Self. Au contraire, quand il y a d'éventuelles résistances qui bloquent l'évolution du processus d'individuation, la tendance sera à la souffrance (stress nerveux), à la maladie psychique et à une forme handicapée de l'être humain, car l'inconscient essaie de compenser l'unilatéralité de l'individu à travers le principe de l'« enantiodromie » 3 .

Selon Jung, les individus privilégient l'un ou l'autre type d'adaptation. Cependant, il considère que l'adaptation n'est pas suffisante pour obtenir le processus

2. A. Houaiss et M. Villar, Dicionário Houaiss de Língua Portuguesa. Lisboa, Círculo de Leitores, 2001, p. 2083.

3. Selon Jung, l'enantiodromie se produit chaque fois qu'il y a une tendance unilatérale de contrôle par le conscient. Plus tard, le processus se déroule dans la direction opposée. Jung utilise l'enantiodromie pour expliquer ce qui s'est passé, par exemple, avec Nietzsche et saint Paul. Selon Jung, les deux ont été touchés par des positions extrêmes dans la vie. Nietzsche, qui était l'anti-Christ, s'identifie avec le Christ à la fin de sa vie. Saint Paul s'est converti au christianisme, après avoir été l'opposé (J. R. Staude, O Desenvolvimento Adulto de C. G. Jung. São Paulo, Cultrix, 1981, p. 104). 
d'individuation. Le Self-l'archétype de la totalité ou le centre régulateur de la psyché - a un pouvoir trans-personnel, une force qui transcende l'Ego et sur lequel l'Ego ne possède pas un contrôle total. Celui-ci permet une harmonisation et une intégration entre deux voies en permanence désarticulées de la psyché humaine : la voie de la conscience, qui protège la raison et la cohérence ; et la voie chaotique de l'inconscience, qui frise les limites du supportable 4 .

Le Self peut être envisagé, si on veut, comme un Dieu intérieur parce qu'il nous pousse vers la plénitude, vers notre totalité, vers l'intégration de nous-mêmes avec notre propre ombre, vers nos volontés, nos motivations et nos projections et vers la reconnaissance authentique de qui nous sommes et de ce qui est réellement important pour notre histoire individuelle. L'individuation est, ainsi, un processus archétypique qui permet l'apparition lente d'une personnalité chaque fois plus ample qui n'est ni bloquée par le côté social (le masque) ni par le côté de l'ombre et de l'inconscient qui nous pousse parfois à l'action destructive de l'autre. Autrement dit, nous pouvons affirmer que ce qui symbolise, pour Jung, le processus d'individuation, c'est la relation excentrique entre le côté conscient et le côté inconscient de la psyché humaine. Paraphrasant les idées de Pascal Chabot ${ }^{5}$, l'individu n'est pas une substance évolutive linéaire bien délimitée mais plutôt le résultat d'un processus excentrique et tendu d'individuation. Ce processus, comme le dit Jung, se développe en forme de spirale, d'une façon contradictoire.

Le processus d'individuation a néanmoins besoin de l'ego (je-conscient). Selon Jung, l'ego est fondamental à la finalisation du processus d'individuation parce qu'il essaie d'orienter un peu plus consciemment l'interprétation du Self en lui attribuant un sens plus pratique. C'est précisément celle-ci l'essence de l'ego pour Jung. Et c'est pour cela que l'ego a une dimension éthique. Une fois que le Self représente toute la gamme des phénomènes de la psyché, qui comprend le conscient (ego) et l'inconscient, l'ego devient le centre de la sphère de la conscience 6.

Le processus d'individuation de Jung ne se réduit pas à une théorie psychologique, il est une théorie sociale de l'éthique. Le caractère autorégulateur de l'individu est transversal au processus d'individuation de Jung 7.

À partir de la "fonction transcendante ", Jung explique aussi que l'échange dynamique entre les pôles de la personnalité, qui vont des contenus du côté conscient jusqu'aux contenus du côté inconscient, permet la transition d'une attitude vers l'autre. C'est-à-dire que les transferts d'un pôle de la personnalité vers l'autre sont gérés par des processus d'individuation, qui sont le résultat du mélange du conscient et de l'inconscient 8 .

4. C.G. Jung, Two Essays in Analytical Psychology. London, Routledge, 1966, p. 244.

5. P. Chabot, La philosophie de Simondon. Paris, Vrin, 2003, p. 75.

6. J.R., Staude, O Desenvolvimento Adulto de C. G. Jung, op. cit., pp. 109-111.

7. Ibid., p. 99.

8. Ibidem, p.100. 
Jung considère aussi les contenus archétypes - bien exposés dans les tragédies grecques et dans les mythes des sociétés dites pré-modernes qui survivent dans l'imaginaire des nouveaux media et du cinéma - comme fondamentaux pour la construction de la psyché. Les archétypes sont considérés comme des images primordiales héritées et sans contenus prédéterminés. Ils sont vides et susceptibles d'être remplis par le matériel provenant de l'expérience consciente de l'individu. D'une autre manière, nous pouvons dire que les images archétypiques sont remplies de contenus qui sont le résultat d'un processus d'individuation qui dépend de la manière, à la fois contradictoire et peu linéaire, dont nous vivons (et essayons de gérer) les situations de notre personnalité, du contexte historique, de notre histoire de vie et de beaucoup d'autres facteurs 9 .

Cependant, deux dangers sont associés à une identification désordonnée avec les archétypes : 1) un hypnotisme généré par les connaissances, surtout par celles qui semblent capables d'expliquer presque tout l'univers ; 2) en absorbant la connaissance et, par conséquence, en élargissant la conscience, l'individu atteint un niveau non humain, s'éloignant des autres. Jung désigne cet effet comme l'« effet de l'inflation » qu'il faut gérer. L'inflation est « une expansion de la personnalité qui va au-delà de ses propres limites, par l'identification avec un archétype ou avec la personne » 10 . En quelque sorte, nous constatons la présence d'une notion amplifiée d'individuation qui signale une liaison différente avec le monde humain et non humain qui nous entoure dans la société postmoderne.

L'ego est aussi fondamental dans le processus d'individuation, il est la voix consciente et rationnelle au moment de prendre une décision 11. C'est pour cela que le processus d'individuation implique d'un côté des décisions éthiques et, de l'autre, des volontés et des désirs. L'éthique est le fil conducteur de l'ego, alors que le désir et la volonté sont les guides de l'inconscient. Jung considère que cette éthique est fondamentale pour la compréhension des archétypes inconscients, car "leur incompréhension, ainsi que l'absence de sens de la responsabilité éthique, privent l'existence de sa totalité et confèrent à beaucoup de vies individuelles une étampe de douloureuse fragmentation " 12 à l'image de ce que nous vivons dans la crise des sociétés modernes avec l'émergence de nouvelles liaisons.

\section{L'individuation chez Gilbert Simondon}

Même si la notion de Jung concernant le développement de l'être humain est très intéressante et importante pour la compréhension actuelle des phénomènes des nouvelles liaisons (ou médiations) humaines, nous considérons qu'il serait bénéfique d'adopter une autre perspective qui rehausse une question superficiellement

9. C.G., Jung Memórias, sonhos, reflexões. São Paulo, Editora Nova Fronteira, 1989.

10. C.G., Jung The Development of Personality. London, Routledge, 1964, p. 356.

11. J.R., Staude, O Desenvolvimento Adulto de C. G. Jung, op. cit.

12. C.G., Jung Memórias, sonhos, reflexões. São Paulo, Editora Nova Fronteira, 1989, p. 171. 
envisagée par Jung : la question du processus qui nous produit comme sujets, comme « individus » dans la liaison avec le monde non humain comme, par exemple, avec les objets techniques.

En ce qui concerne l'individuation, Jung privilégiait le regard tourné vers l'intérieur. Un regard proche du sujet cartésien. Du fait d'avoir une vision très interne du processus, marquée par la pratique de la psychanalyse, Jung avait tendance à exclure un peu le monde extérieur 13. Le Self serait, par-dessus tout, un produit intérieur à l'individu, les archétypes sociaux constituant son champ d'analyse privilégié. En vertu de sa formation de base, Jung oublie un peu les liaisons avec le plan extérieur. Staude suggère même que son sens aigu de la culture et de l'histoire était fabuleux. Cependant, «sa compréhension et son intérêt pour la structure sociale, pour les institutions sociales et pour la toile de relations sociales étaient [...] peu développés »14. En prolongeant cette vision de Staude, nous pouvons dire que Jung a peu développé la question de l'individuation dans les liaisons que l'être humain établit avec les objets techniques en général.

En accord avec les raisons déjà citées, nous trouvons chez Gilbert Simondon un complément qui peut perfectionner la pensée autour du processus d'individuation. La pensée de Simondon souligne l'idée qu'il n'y a pas une unité humaine stable, qu'il n'existe pas d'individu finalisé. Il existe, avant tout, des individus en processus permanent d'individuation, où ils sont toujours en train de s'in-diviser et de se diviser.

Simondon propose une vision originale de l'individuation. En premier lieu, son approche établit une certaine rupture avec la philosophie traditionnelle $e^{15}$. S'il est vrai que les archétypes, chez Jung, signifient les formes immatérielles dans lesquelles les phénomènes psychiques (les contenus) tendent à se mouler, correspondant aux modèles innés qui servent de matrice pour le développement de la psyché, pour Simondon celle-ci est une espèce de système en tension où le processus d'individuation se développe d'une façon complexe et non linéaire. Pour Simondon, les idées ne sont pas les grands guides de l'action. Leurs contenus sont à peine des accessoires pour certaines situations 16 .

En deuxième lieu, Simondon avait une vision un peu différente de Jung au sujet de la question de l'objectif final de l'individuation. Dans une citation claire qui montre sa vision par rapport à l'univocité de l'être, Simondon soutient que "pour penser l'individuation, il est nécessaire de considérer l'être non comme une substance ou matière, ou une forme, mais comme un système tendu, supersaturé, audessus du niveau de l'unité, ne consistant pas seulement par soi-même, et ne s'accordant pas à la réflexion à travers le principe du tiers exclu ; l'être concret ou l'être complet, c'est-à-dire l'être pré-individuel, c'est un être qui est plus que l'unité »17.

13. J.R., Staude, O Desenvolvimento Adulto de C. G. Jung, op. cit., p. 129.

14. Ibid.

15. P. Chabot, La philosophie de Simondon, op.cit., p. 107.

16. Ibid., p. 111.

17. G., Simondon, L'individuation psychique et collective. Paris, Aubier, 1989, p. 13. 
On pourra quand même dire que la conscience (la perception altérée) de l'être préindividuel (voir l'influence de Merleau-Ponty sur Simondon) est, d'une certaine façon, une conscience amplifiée, un domaine élargi de l'individuation au sens de Jung.

Pour Simondon, " une telle individuation ce n'est pas la rencontre d'une forme et d'une matière préliminaire existant comme des termes séparés déjà constitués, mais une résolution qui surgit dans la piste d'un système méta-riche de potentiels: forme, matière et énergie préexistantes dans le système [...]. Le vrai principe d'individuation est la médiation et non l'individu ou l'élément singulier » 18 . On peut dire que forme, matière et énergie préexistantes dans le système sont un peu les archétypes chez Jung. Pour Simondon, l'action thérapeutique (le changement) n'est pas centrale, comme chez Jung, mais elle est présente dans sa réflexion théorique. On dirait que la conscience de l'univocité de l'être (voir Deleuze et Parménide) et de l'individuation comme changement de perception peut fonctionner comme une thérapie jungienne. Ainsi, Simondon est, pour l'essentiel, d'accord avec Jung mais avec différentes intentions et selon différents contextes.

Il y a plusieurs aspects en commun entre Jung et Simondon. Un de ces aspects, c'est le regard constant à l'égard du processus intermédiaire de l'individuation. Dans le cas de Jung, c'était un regard qui portait sur les relations complexes entre les archétypes (comme formes vides) et les niveaux du processus en spirale d'individuation. Selon Simondon, la perspective est plus philosophique, bâtie sur un principe d'analyse de base ontologique dans la mesure où les processus internes entre le self et le "Je » peuvent être appliqués aux liaisons entre humains et objets techniques, surtout à ceux qui possèdent un haut degré de complexité ou de concrétisation (voir les nouvelles liaisons avec support digital).

Ainsi, Jung cherchait à saisir en soi-même (et chez ses patients), inspiré par les religions orientales (voir ses études sur le Bouddhisme), l'être individué, équilibré et ouvert au monde non-humain, qui serait le supposé résultat espéré de la guérison. C'est pour cela que Jung tendait à envisager l'individuation dans un sens un peu hylémorphique et quelquefois centré dans l'individu (voir l'évolution du concept dans son œuvre). Dans une de ses vastes références à l'individuation, Jung disait que l'individuation était un " processus à travers lequel nous devenons ce qu'effectivement nous sommes. L'individuation permet une progressive intégration du Self inconscient dans la vie de l'individu en accord avec ses limites de temps et d'espace ». 19

Simondon s'est inspiré, pour sa part, des études du paléoanthropologue LeroiGourhan, qui considérait l'individuation sous le prisme de l'ontogénie dans une vision non anthropocentriste. Par rapport à ce refus de regarder l'être comme quelque chose de fini, comme une synthèse, Deleuze affirme que "Simondon insiste sur cette espèce de dimension qui n'est pas du tout une synthèse, et on ne peut pas

18. Ibid., p. 16.

19. C.G. Jung, Two Essays in Analytical Psychology, op. cit., p. 171. 
dire que cet intermédiaire [la zone obscure] est une synthèse. [...] C'est en effet une terre inconnue $» 20$.

\section{Conclusion : le concept d'individuation et la compréhension de la société postmoderne}

"Tous les Romains étaient encerclés par les esclaves. L'esclave et sa psychologie ont envahi l'Italie antique et tous les Romains intérieurement

- et bien sûr inconsciemment - sont devenus des esclaves. Vivre en permanence dans une atmosphère d'esclaves a affecté leur psychologie, à travers l'inconscient. Personne ne peut empêcher une telle influence. "

C. G. Jung

Pour Jung et Simondon, la question de l'individuation aborde la complexité des rapports des humains avec eux-mêmes et avec leur environnement. Le cas des esclaves des Romains illustre la notion fondamentale de Jung : nous sommes une membrane perméable à tout ce qui nous entoure 21 . Si les formes archétypales de la société sont fondées sur la domination totale (l'esclavagisme), ce fait conditionne la façon dont nous gérons nos relations entre l'organisme de coordination, lié à notre néocortex, et notre Self marqué par les archétypes de l'inconscient collectif qui hérite, d'une façon active, des formes extérieures. Pour Jung, cette membrane qui nous entoure renvoie davantage à un pont qu'à des portes séparées.

De ce point de vue, les approches hétérodoxes de la théorie de Simondon ont également refusé la notion occidentale de l'individu. L'humanité est comme un système métastable par rapport à l'environnement technique et social qui l'entoure (une sorte d'ethno-techno).

Ainsi, la conclusion de Jung suivant laquelle tous les Romains deviennent intérieurement - et bien sûr un peu inconsciemment - des " esclaves " devient très claire : la relation avec ces deux instances se développe comme un virus pour le maître d'esclaves (et aussi dans les relations hommes et femmes) et vice-versa. Il y a une création d'une société où le virus du social est basé sur une logique de puissance, de possession d'un autre animal (dans ce cas, humain). La gestion de ces instances intérieures a tendance, comme un virus ou une épidémie, à parcourir l'ensemble du tissu social tissé par cette énergie de l'ombre, comme disait Jung.

20. G. Deleuze «Anti-Edipe et Mille Plateaux. Les codes ; Le capitalisme ; Les flux ; Capitalisme et schizophrénie ; La psychanalyse »1971, en ligne : http://textz.gnutenberg.net/ textz/deleuze_gilles_anti-oedipe_et_mille_plateaux.txt. La tentative de Gilles Deleuze de penser les différents modes d'individuation biopsychique est due à l'influence des travaux de Gilbert Simondon (cf. Combes, 1999, p. 6).

21. Cf. M. Maffesoli, L'instant éternel. Paris, La Table Ronde, 2000, trad. portugaise $O$ Eterno Instante. O Retorno do Trágico nas Sociedades Pós-Modernas. Lisboa, Instituto Piaget, 2001. 
La même logique anime les réseaux techno-sociaux de la postmodernité d'aujourd'hui (voir l'importance des réseaux comme Facebook dans le quotidien de millions des personnes).

Prenons un exemple concret : "Dans le contexte de ma pratique clinique, un patient, je vais l'appeler Paul, est un professeur d'université de 30 ans, marié et sans enfant. Il a commencé une analyse (thérapie) du fait de se plaindre d'anxiété chronique et d'insomnie. Il enseigne dans une université privée bien connue à Rio de Janeiro. Paul a commencé à travailler dans cet établissement après avoir terminé sa maîtrise, c'est-à-dire depuis six ans. À ce jour, cependant, il n'a pas encore de contrat officiel de travail et, par conséquent, il ne bénéficie pas des droits des travailleurs. Ses cours durent généralement deux mois. À la fin de chaque période scolaire, l'anxiété augmente et il est affecté par des crises d'insomnie. Paul ne sait jamais s'il va continuer avec la même charge de travail ou, pire encore, il ne sait jamais si, à la fin du semestre, il sera recruté dans l'enseignement, c'est-à-dire qu'il ne sait jamais avec certitude s'il aura du travail. En effet, il y a eu des périodes où l'université ne lui a pas proposé de nouveaux cours. La justification des employeurs? Aucune. Son désespoir n'est pas plus grand parce qu'il est employé dans un autre établissement d'enseignement dans ce domaine, avec un contrat formel. L'incertitude au sujet de son occupation et, par conséquent, l'incertitude d'avoir de l'argent pour payer les factures à la fin du mois, ont affecté ses relations avec sa femme, qui est aussi une professionnelle, qui souffre de la même instabilité financière. Comment faire des plans à long terme - tels qu'avoir des enfants, par exemple - si on est tellement instable? »22.

Les relations antérieurement stables deviennent tendues à cause d'un système de contrat de travail flexible dans lequel l'ancien contrat stable disparait peu à peu. Dans ce contexte, il y a une épidémie qui infecte et affecte tout l'imaginaire, l'esprit du temps. Prenez, par exemple, le travail immatériel qui se déroule de plus en plus sur Internet avec le courrier électronique, les réseaux sociaux écoutant de la musique sur YouTube, jouant aux jeux vidéo dans une accélération constante. L'individuation antérieure basée sur un lien entre la sécurité et l'occupation régulière et quotidienne de l'espace et du temps (le temps du travail matériel du monde taylorien) tend à disparaitre progressivement. Dans ce contexte, l'Internet et ses liens décontextualisés et sans interaction face à face, comme dans les réseaux sociaux digitaux, ont un rôle décisif dans l'imaginaire postmoderne ${ }^{23}$ et dans la transformation de la notion d'individu, de sujet. Comme dit Maffesoli 24 , « la personne postmoderne, confrontée au destin, ne peut pas être limitée au petit je produit par la

22. H. Pereira, "Da Metamorfose dos Deuses: Capitalismo, Psique e Sintoma no Século XXI", en ligne : http://www.himma.psc.br/artigos/artigo_06_2k8.htm (consulté le 3 février 2010).

23. M. de Lemos Martins, "La nouvelle érotique interactive », Sociétés, n 96, 2007, pp. 21-27.

24. M. Maffesoli, op. cit., p. 192 
crispation égocentrique qui fut la marque des deux ou trois siècles qui viennent de s'écouler ».

Avec le capitalisme néolibéral et l'émergence de la société postmoderne, il y a comme un air du temps, un imaginaire, comme soutient Maffesoli, une image qui agit comme une épidémie qui nous englobe tous et qui peut augmenter avec les nouvelles formes chaotiques et anomiques de vivre la vie quotidienne dans la société postmoderne.

Il y a une nouvelle forme d'individuation, qui invite à sortir de soi. En effet, « il y a l'élément de se perdre à partir de soi ou sortir de soi. L'ex-stasi, comme dit Baudrillard, est de l'ordre de l'éclatement: donc "on va s'éclater" dans YouTube, ou dans Second Life, pour citer des exemples dont le Net naturellement est riche » 25. Dans ce contexte, il faut résister, découvrir une thérapie qui fait appel à l'amitié et à la fusion communautaire et paléopolitique 26 , une forme d'individuation plus pleine, comme disait Jung, ou pré-individuelle, comme disait Simondon.

\section{Bibliographie}

Chabot P., La philosophie de Simondon. Paris, Vrin, 2003.

Deleuze G., «Anti-Edipe et Mille Plateaux. Les codes ; Le capitalisme ; Les flux ; Capitalisme et schizophrénie ; La psychanalyse »1971, en ligne : http://textz.gnutenberg.net/ textz/deleuze_gilles_anti-oedipe_et_mille_plateaux.txt.

Houaiss A. et Villar M., Dicionário Houaiss de Língua Portuguesa. Lisboa, Círculo de Leitores, 2001.

Jung C.G., "Simbolismo e Transformação na missa », in Read, H., Fordham, M., Adler G., McGuire W. et Hull, C., Psicologia e Religião: Ocidente e Oriente. São Paulo, Cultrix, 1958.

Jung C.G., Memórias, sonhos, reflexões. São Paulo, Editora Nova Fronteira, 1989.

Jung C.G., The Development of Personality. London, Routledge, 1964.

Jung C.G., Two Essays in Analytical Psychology. London, Routledge, 1966.

La Rocca F., Nouvelles technologies et construction sociale de l'hyperréalité, en ligne : http://www.ceaq-sorbonne.org/node.php?id=97\&elementid=900 (consulté le 1er juillet 2007).

Lash S. et Urry J., Economies of Signs and Space. London, Sage Publications, 1994.

Martins M.L. (2007), «La nouvelle érotique interactive », Sociétés, nº 96, 2007, pp. 21-27.

Maffesoli M., L'instant éternel. Paris, La Table Ronde, 2000, trad. portugaise O Eterno Instante. O Retorno do Trágico nas Sociedades Pós-Modernas. Lisboa, Instituto Piaget, 2001.

McLuhan M., Os meios de comunicação como extensões do homem. São Paulo, Cultrix, 1974.

Miranda J.A.B. et Cruz M.T. (Eds.), Crítica das ligações na Era da Técnica. Lisboa, Tropismos.

25. F. La Rocca, "Nouvelles technologies et construction sociale de l'hyperréalité », en ligne : http://www.ceaq-sorbonne.org/node.php?id=97\&elementid=900 (consulté le 1er juillet 2007).

26. P. Sloterdijk, Dans le même bateau : essai sur l'hyperpolitique. Paris, Rivages, 1997. 
Neves J.P., O Apelo do Objecto Técnico. Porto, Campo das Letras, 2006.

Neves J.P. et Pedro Costa P.R., " Algumas notas sobre o conceito de individuação em Jung e Simondon: pensando a natureza das novas mediações técnicas ", in Congresso LUSOCOM, Universidade Lusófona, Lisboa, 2009.

Pereira H., « Da Metamorfose dos Deuses: Capitalismo, Psique e Sintoma no Século XXI », en ligne : http://www.himma.psc.br/artigos/artigo_06_2k8.htm (consulté le 3 février 2010).

Read H., Fordham M., Adler G., McGuire W. et Hull C., Psicologia e Religião: Ocidente e Oriente. São Paulo, Cultrix.

Simondon G., L'individuation psychique et collective. Paris, Aubier, 1989.

Sloterdijk P., Dans le même bateau : essai sur l'hyperpolitique. Paris, Rivages, 1997.

Staude J.R., O Desenvolvimento Adulto de C. G. Jung. São Paulo, Cultrix, 1981. 